\title{
Effect of Color Output Modification Approach (COMA) on the gray balance: A mismatch of device calibration, destination and source profiles, and halftone screening
}

\section{ABSTRACT}

The purpose of this applied research in the digital print production was to determine the influence of applied Color Output Modification Approaches (COMA) or Color Output Sequences (COS) to determine the colorimetric variations (COLVA) on the gray balance (GB) hue in a Color Managed Digital Printing Workflow (CMDPW). The experiment analyzed the application of eight COMA or COS on the digital color output. The data contained in this report are the result of an experiment conducted to test an accepted color management practice to gain a greater understanding of the presumptions associated with the application of correct print parameters (Calibration, Screening, Resolution, Source and Destination Profiles) of CMDPW. These parameters rely on the 4-C's (Calibration, Characterization, Conversion and Control) of Color Managed Workflow (CMW). The experiment examined the eight COMA as independent groups $(K=8)$ using a one-way Analysis of Variance (ANOVA) with equal $n$ 's method (at $\alpha=0.05$ ) to determine the significant colorimetric variations that exists among the $[K=8, N=$ $\left.800, n_{i}=80, n_{t}=640\right]$ groups gray balance average hue deviations. With eight groups, a one-tailed, non-directional hypothesis was established. The conclusions of this study are based upon an analysis of the COLVA by ANOVA test data and associated findings. The data from the ANOVA revealed significant differences in the COLVA of the reproduction among the multiple COMA groups. The colorimetric data derived by the experiment suggest that selection of correct print parameters is an important step in a CMDPW as it relates to achieving accurate GB for a desired use/purpose.
Haji Naik Dharavath (1)

Graphics Technology

Central Connecticut State University New Britain, USA

Corresponding author: Haji Naik Dharavath e-mail:dharavathh@ccsu.edu

First recieved: 23.12.2019. Accepted: 07.04.2020.

\section{KEY WORDS}

Calibration, color, colorimetry, gamut, profiling, screening

\section{Introduction}

Analyzing the color image by examining its quantitative attributes eliminates the subjective judgment on color quality evaluation of printed colors or colors in nature. Advancements in science and engineering, however, have allowed print and graphic professionals to apply scientific research methods across printing workflow. Applying these methods heightens the importance of proper print production workflow. Workflow is represented through schematic illustrations of activities that reflect the systematic organization of analog and digital devices used during the print and image production process. A print ready e-file (.PDF or .JPEG or .PSD or PostScript, etc.) is likely to be manipulated and later printed by an array of output digital devices [computer-to-plate (CTP), digital printers and printing presses]. Given each family of devices tends to create and produce color differently, 
the challenge is to manage color consistency across the entire workflow. Analysis of color image quality in the commercial printing workflow requires a Color Management System (CMS) to assist the color producer (printer operator or the designer) in delivering accurate output colors regardless of device color capacities with the use of proper color management techniques (see Figure 1).

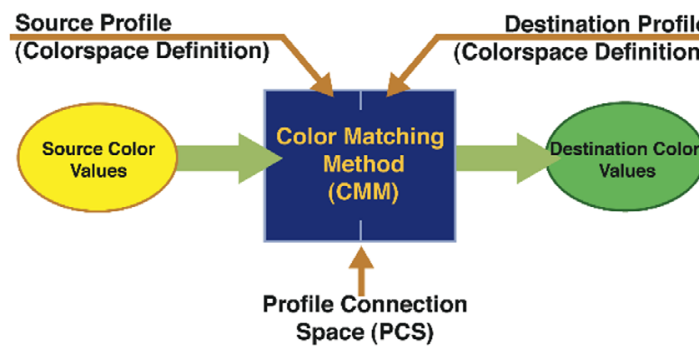

» Figure 1: Schematic of PCS of CMS (Retrieved from
https://www.adobe.com/)

Digital color print reproduction involves physical/ mechanical interaction among the imaging cylinder, dry/ liquid toner, and the substrate (Novaković \& Avramović, 2012). The outcome of this interaction is the color print. Color can be viewed as a science where the optical aspects of color are quantitatively analyzable and measurable. The human eye, however, perceives color more subjectively, which poses a challenge at times for the printing and image reproduction industry. A continuous-tone color image is composed of a full spectrum of shades and color, from near white to dense black. In a traditional printing (offset, digital offset, gravure or flexography) workflow, the method by which continuous-tone photographic images are transformed to a printable image is called halftoning. In this method, varying percentages of the printed sheet are covered with halftone dots to represent the varying tones in the image. The ink (paste or liquid ink) printed by each dot, of course, has the same density. At normal viewing distance, the dots of a printed image create an optical illusion of a continuous tone image. In contrast, a simple digital image could be a binary picture, $[\mathrm{h}(\mathrm{x}$, $y)$ ], with each point being either completely black or completely white (Pnueli \& Bruckstein, 1996). A digital halftone is a pixel map, with bit depth, that gives the impression of an image containing a range of gray shades or continuous tones. An 8-bit grayscale image contains 256 different levels of gray from white to black.

Gray balance is the proper percentage of combinations of cyan, magenta, and yellow inks that produce neutral shades of gray. Hue shifts will occur when there is any imbalance of one of the components. The imbalance is due in large part to ink impurities, chromaticity deviation of the substrates, and other attributes. Gray balance is a significant factor in determining overall color gamut. Gray balance can be determined by careful evaluation of a full set of tint charts printed with process inks. Col- orimetric methods are used to determine if the hue of gray is desirable in order to make sure that the black ink scale is neutral. ISO 12647-7 document states that the gray balance can be printed and measured at the CMY overlap (overlap of $\mathrm{C}=50 \%, \mathrm{M}=40 \%$, and $\mathrm{Y}=$ $40 \%$ ). The deviation can be determined from the calculation of $\Delta \mathrm{H}^{*}$ (deviation of hue, $\mathrm{h}^{*}$ ) or $\Delta \mathrm{C}^{*}$ (deviation of chroma, $c^{*}$ ) and it requires the colorimetric data of CMY overlap printing from the $L^{*} a^{*} b^{*}$ model.

Regardless what experts (published reports) say, there is no single/fixed screening method that would work for every color reproduction. Since the introduction of digital workflow (computer to film, to plate, and to press) in the industry, there are many experts who have come up with technologies and tools for color images that would benefit from improved color reproduction technologies. Screening is only an option of such technologies. In the digital printing environment, screening software can create the digital version of the amplitude modulate (AM) and also of the frequency modulated (FM) halftone screen. Screening software in the raster image processor (RIP) of the color managed workflow (CMW) at a digital printing press front-end platform (DFE) applies a digital dot pattern to the color image during printing.

The quality of color image reproduction of any type of printing (digital or traditional) is largely influenced by the properties of paper. Paper is considered a commodity, but its properties are a long way from being standardized (Wales, 2009). In order to produce quality printed materials (a good halftone image), the press operator must carefully manage several print parameters, such as the source colors (a source profile of ISO/ANSI standard), press calibration, press characterization (device destination profile), and the screening option. Without assigning these parameters to a print job would result in a color mismatch.

\section{Amplitude Modulated (AM) Screening}

In AM screening, the dot frequency is constant and dot amplitude varies. AM screening breaks the image into dots of varying sizes, which are clustered together, at a fixed screen angle. For each color (CMYK) to be overprinted, the halftone CMYK separations need to be generated at a particular screen angle to avoid a moiré pattern. The dot is generated from a cluster of laser spots. A halftone cell (or grid) is then divided into a matrix of single recording dots (laser spots). Since there is always a physical and mathematical relationship among the number of gray levels, the resolution of the output device, and the screen ruling, there is always a tradeoff between resolution and the number of reproducible gray levels (Fleming, Dollak \& Fryzlewicz, 2008). The number of laser spots within a halftone cell depends on the following parameters: screen frequencies/screen angles, imagesetter resolution/ 
gray levels, dot size, and dot shape/dot patterns. The outcome of the clustering is a continuous tone pattern - lighter or darker shades or tones (See Figure 2).

\section{Frequency Modulated (FM) Screening}

In FM screening, the dot frequency varies and dot size is constant (Ma, 2003). FM screening, also referred to as stochastic screening, can be regarded as an electronic implementation of screenless printing (Chung \& Ma, 1995). The word stochastic means "involving a random variable" - it uses randomly placed dots instead of AM halftone dots aligned along the screen angle. FM screening uses a microdot placement (See Figure 2). The dot size is fixed, but the number of dots within a halftone cell varies according to the tonal value being imaged (Bouman \& He, 2004). The minimum size of the dots is limited only by the output device resolution and dots are measured in microns $(\mu \mathrm{m})$. A typical modern 2400 DPI output device has a laser spot size of 10.6 $\mu \mathrm{m}$ (microns, $10^{-6}$ ) and the size of a microdot is $21 \mu \mathrm{m}$ (Chung \& Ma, 1995). In FM screening, screen angles are not required when generating a CMYK separation. This results in the eradication of the moire pattern in the printed image (Fleming, Dollak \& Fryzlewicz, 2008). FM screening, though, uses smaller dots that are not restricted to a fixed grid pattern. By varying the number of dots in a given area, any desired gray level can be generated. The dots are smaller than the AM screening, therefore FM screening can represent more detail and support higher resolution printing (See Figure 2).

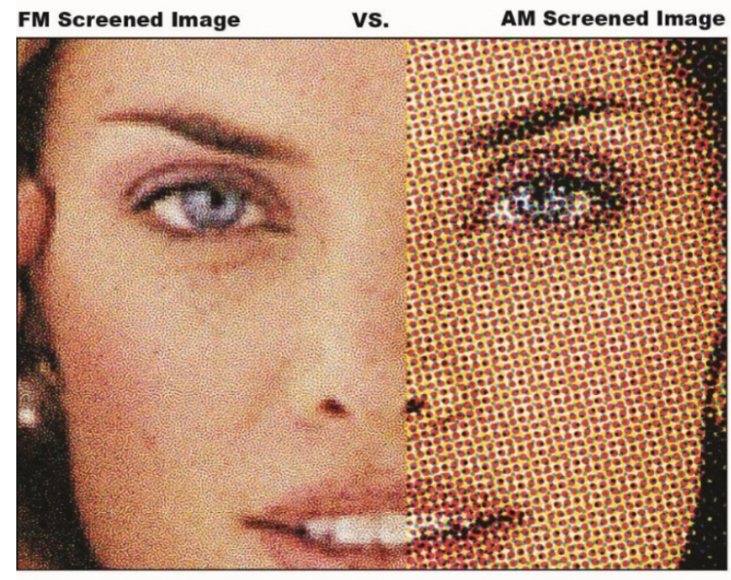

» Figure 2: FM vs. AM screened image (Retrieved from https://www.kymc.com/)

\section{Purpose of the research}

The purpose of this experimental study was to determine the significant differences that exist in the gray hue (overlap of CMY) deviation $(\Delta \mathrm{H})$ among the eight color output modification approaches (COMA) or Color Output Sequence (COS). The experiment was conducted in a color managed digital printing workflow (CMDPW) to determine the effect (impact or influence) of COMA on the printed gray hue based on the statistical evaluation among eight (groups, $\mathrm{K}=8$ ) different types of color print modifications. The following one-tailed non-directional hypothesis was established for this study because of the eight independent COMA groups. A one-tailed non-directional hypothesis predicts the nature of the effect of the independent group on the dependent variable (or the color attribute as the outcome). A non-directional alternative hypothesis does not state the direction of the difference, but indicates only that a difference exists. The one-tailed test provides more power to detect an effect in one direction by not testing the effect in the other direction.

\section{Hypothesis (Gray Colour Hue Deviation)}

$\mathbf{H}_{\mathbf{0}}$ : There is no significant difference (or relationship) in the color printing attribute of Gray $\triangle \mathrm{H}$ (CMY overlap) among the eight COMA when the printed colorimetry is compared against the reference colorimetry.

$\mathbf{H}_{\mathrm{a}}$ : There is a significant difference (or relationship) in the color printing attribute of Gray $\Delta \mathrm{H}$ (CMY overlap) among the eight COMA when the printed colorimetry is compared against the reference colorimetry.

\section{Limitations of the Research}

For this experiment, there were limitations to the technology used within the graphics program laboratory. Prior to printing and measuring the samples, the digital color output printing device and color measuring instruments (spectrophotometer and densitometer) were calibrated against the recommended reference. The print condition associated with this experiment was characterized by, but not restricted to, inherent limitations: colored images (TC1617x, ISO300, and ISO12647-7) chosen for printing, desired rendering intent applied, type of digital printer for proofing/printing, type of paper for printing, type of toner, resolution, screening technique, use of defined color output profiles, calibration data applied, and so on. Several variables affected the facsimile reproduction of color images in the CMDPW and most of them were mutually dependent. The scope of the research was limited to the color laser (electrophotographic) digital printing system (printing proof/printing) and other raw materials and the multiple types of color measuring devices and color management and control applications (data collection, data analysis, profile creation, and profile inspection) used at the university graphics laboratory. Findings were not expected to be generalizable to other CMDPW environments. It is quite likely, however, that others could find the method used and the data of this article meaningful and useful. The research methodology, experimental design, and statistical analysis 
were selected to align with the purpose of the research, taking into account the aforementioned limitations.

\section{Research methodology}

The digital color printing device used in this experiment was a Konica-Minolta bizHub C6000 Digital Color Press. It uses a Creo IC-307 raster image process (RIP) application (front-end system). A two-page custom test target image $\left(12^{\prime \prime} \times 18^{\prime \prime}\right.$ size) was created for proofing and printing use for the experiment (See Figures 3 \& 3A). The test target contained the following elements: an ISO 300 and generic images for subjective evaluation of color, an ISO 126477 Control Strip (2013, three-tier), and a TC1617x target for gamut/profile creation. Table 1 presents the variables, materials, conditions, and equipment associated with this experiment. Colorimetric, Densitometric, and Spectrophotometric data were extracted by using an X-Rite Eye-One Spectrophotometer and an X-Rite i1iO Scanning Spectrophotometer from the color printed samples for the statistical analysis to determine the significant differences that exist among the eight COMA groups. Print/ Color attributes (primary colors and gray hue) from each group were analyzed/compared with one another. For all the eight COMA groups, a total of 800 samples of target color images were printed, 100 prints for each substrate group, noted by letter " $N$ " $(N=100)$. Of 100 samples of each group, 80 samples were randomly selected from each COMA group, and measured, noted by the letter " $n$ " ( $n=80)$. Glass \& Hopkins (1996) provides an objective method to determine the sample size when the size of the total population is known. The following formula was used to determine the required sample size, which was $80(n)$ printed sheets for each COMA for this study:

$\mathrm{n}=\left[\chi^{2} \mathrm{NP}(1-\mathrm{P})\right] /\left[\mathrm{d}^{2}(\mathrm{~N}-1)+\chi^{2} \mathrm{P}(1-\mathrm{P})\right]$

$\mathrm{n}=$ the required sample size

$\chi^{2}=$ the table value of chi-square for 1 degree of

freedom at the desired confidence level (3.84)

$\mathrm{N}=$ the total population size

$\mathrm{P}=$ the population proportion that it is desired to estimate (.50)

$d=$ the degree of accuracy expresses as a proportion (.05)

\section{Digital Color Press (printer)}

Test target TC1617x image was selected for the output device profile creation process. The target image was placed into an Adobe InDesign-CC layout (12" W x 18" $\mathrm{H})$ and a .PDF file was created without any image/color compression techniques (see Figures 3 \& 3A). Prior to printing the patches/target image, the printer was calibrated for both the screening options (AM and FM) according to its manufacturer specifications. A calibration process means standardizing the performance of the
Table 1

Experimental and Controlled Variables

\begin{tabular}{|c|c|}
\hline Variable & $\begin{array}{l}\text { Material/Condition/ } \\
\text { Equipment }\end{array}$ \\
\hline Test image & Custom Test Target, 2 pages \\
\hline \multirow{2}{*}{ Control strips/targets } & ISO 12647-7 (2009 \\
\hline & \& 2013), TC1617x \\
\hline \multirow{2}{*}{ Other Images } & $\mathrm{B} / \mathrm{W}$ and Color for \\
\hline & Subjective Evaluation \\
\hline Profiling Software & X-Rite i1PROFILER 1.8 \\
\hline Profile Inspection Software & Chromix ColorThink-Pro 3.0 \\
\hline Image Editing Software & Adobe PhotoShop-CC \\
\hline Page Layout Software & Adobe InDesign-CC \\
\hline Source Profile (RGB) & Adobe 1998.icc \\
\hline Destination Profile (CMYK) & Custom, Konica-Minolta.icc \\
\hline Reference/Source & \multirow{2}{*}{ GRACoL2013.icc } \\
\hline Profile (CMYK) & \\
\hline Color Management & \multirow{2}{*}{ Adobe (ACE) CMM } \\
\hline Module (CMM) & \\
\hline Rendering Intents & Absolute \\
\hline Computer \& Monitor & Dell OPTIPLEX/LCD \\
\hline Raster Image Processor (RIP) & Creo IC-307 Print Controller \\
\hline \multirow{2}{*}{ Printer } & Konica-Minolta bizHub \\
\hline & C6000 Color Laser \\
\hline Achieved CMYK SID for all & $C=1.47 ; M=1.37$ \\
\hline print runs (AM vs. FM) & $\mathrm{Y}=0.90 ;$ and $\mathrm{K}=1.79$ \\
\hline Screens and Screen Ruling & AM \& FM, 190 LPI for AM \\
\hline Print Resolution & $600 \times 600 \mathrm{DPI}$ \\
\hline Toner & Konica-Minolta Color Laser \\
\hline \multirow{2}{*}{$\begin{array}{l}\text { Type of Paper Weight/ } \\
\text { thickness }\end{array}$} & Hammermill 100LB Matte \\
\hline & Coated, Sheetfed \\
\hline Type of Illumination/ & $D 5$ \\
\hline Viewing Condition & DSO \\
\hline \multirow{5}{*}{ Color Measurement Device(s) } & X-Rite Eye-One PRO \\
\hline & Spectrophotometer \\
\hline & with Status T, $2^{\circ}$ angle, \\
\hline & and i1iO Scanning \\
\hline & Spectrophotometer \\
\hline Data Collection/ & CGS-ORIS CertifiedWEB \\
\hline Analysis Software & and SPSS \\
\hline
\end{tabular}

devices according to the device manufacturer specifications so that the results of the devices are repeatable. The calibration data (range of CMYK densities) were saved in the calibration lookup tables of the RIP and a calibration curve was created. The calibration curve consists of the maximum printable densities of each color (CMYK) of both the screening techniques (See Figure 4).

In a generic color managed digital printing workflow, digital front-end (DFE) platforms (raster image processor or RIP) of digital printers (or presses) offer opportunities for the user (or press operator) to manipulate the output color quality to meet the expected demand of the customer. In order to print a quality halftone image, the user must carefully manage several print parameters, variables, and attributes which are associated with the digital printing process. 
For this experiment, printer resolution was reduced to 600 dots per inch (DPI) because one screening option (FM screen) in the front-end platform (RIP) was limited to only setting the resolution at $600 \mathrm{DPI}$. This is due to random micro-dot placement, without use of screen angles, for the dot reproduction of FM screen- ing. However, the AM screening option offered the ability to set printer resolution at $600 \mathrm{DPI}, 1200 \mathrm{DPI}$, and $2400 \mathrm{DPI}$. In order to test the screening effect on the COMA only the $600 \mathrm{DPI}$ resolution was selected for both screening technologies to keep the parameters/ variables consistent throughout the experiment.
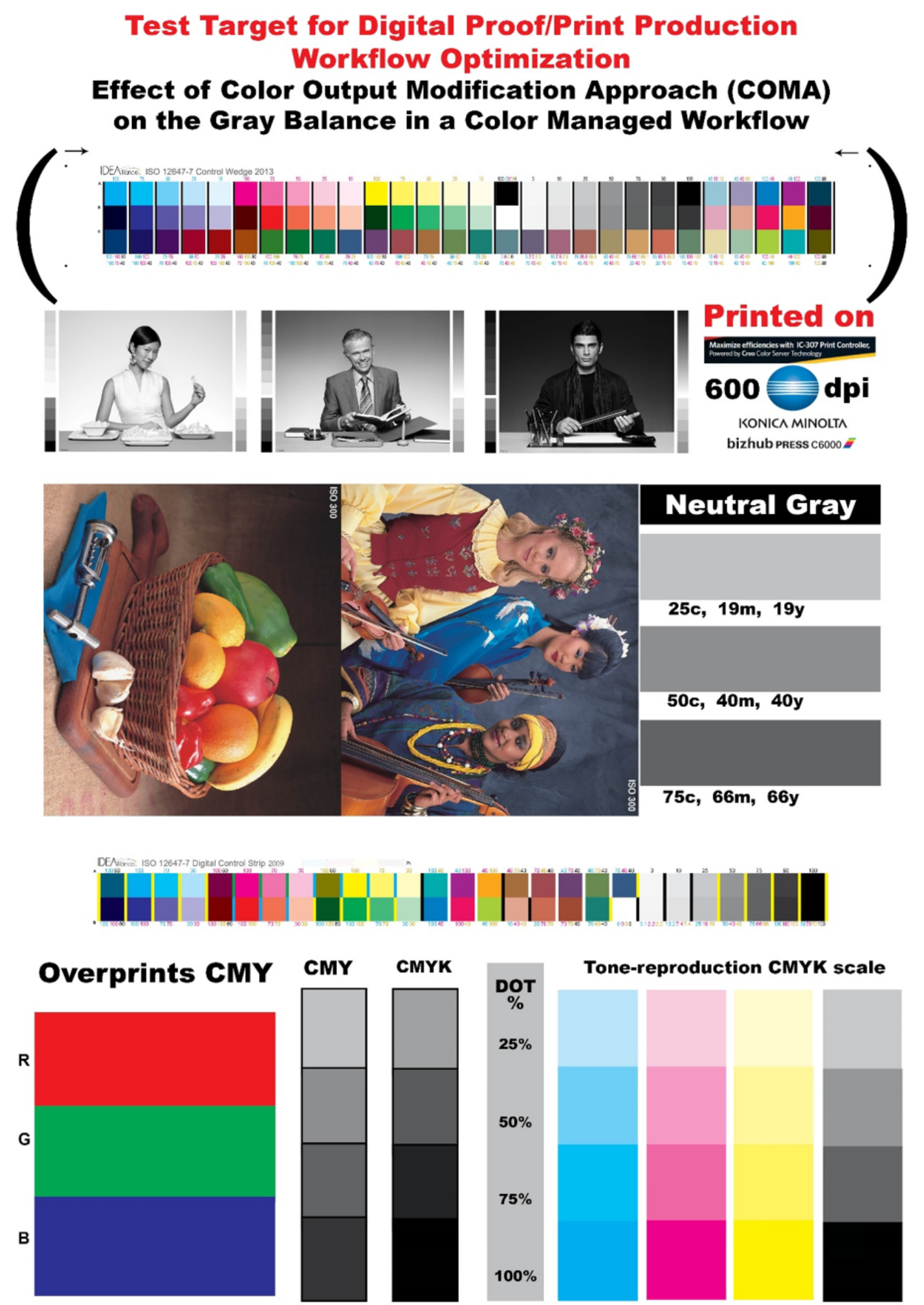

Tone-reproduction CMYK scale

» Figure 3: Test Image for the experiment (PAGE 01) 


\section{Test Target for Digital Proof/Print Production Workflow Optimization \\ Effect of Color Output Modification Approach (COMA) on the Gray Balance in a Color Managed Workflow}

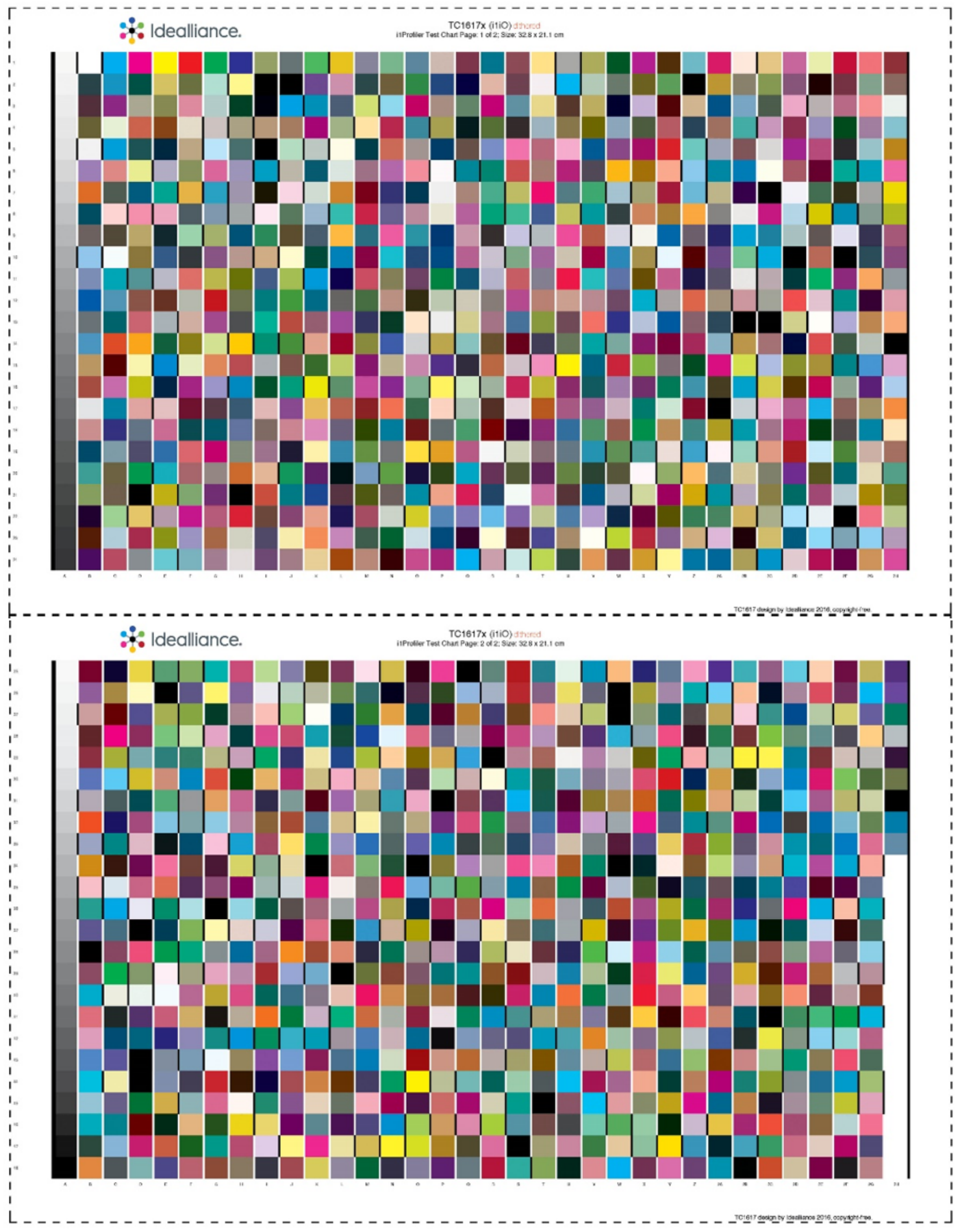

» Figure 3A: Test Image for the experiment (PAGE 02)

\section{Output Device Profile (ODP) with $A M$ and FM Screening}

Hammermill brand, 100 LB matte-coated digital color printing paper $12^{\prime \prime} \mathrm{W} \times 18^{\prime \prime} \mathrm{H}$ was used for printing the samples in the experiment. A total of 100 sheets/copies of TC1617x were printed with the calibration curve attached. Also, an amplitude modulated (AM) halftone screening technique with 190 lines per inch (LPI) and $600 \mathrm{DPI}$ as the printer resolution was applied during the printing. No color management or color correction techniques were applied during the printing. Printed patches of TC1617x were measured in CIE L* a* b* space using the i1PROFILER application with an X-Rite spectro- 


\section{PRINTER CALIBRATION}

\section{AM Screening}

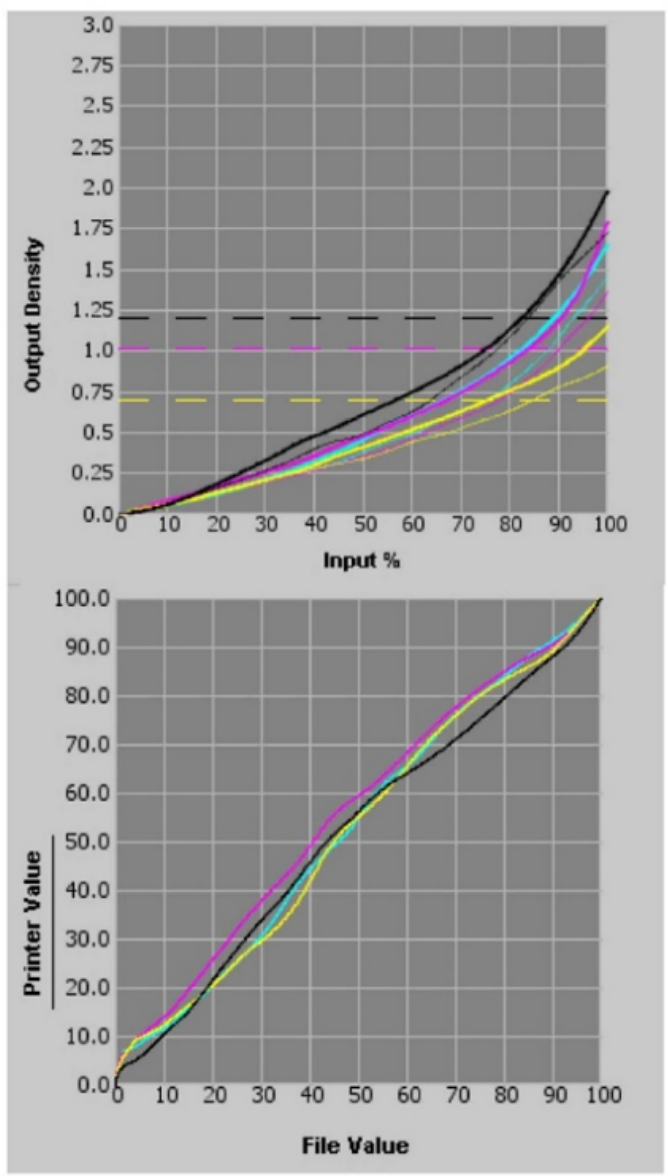

vS.

FM Screening
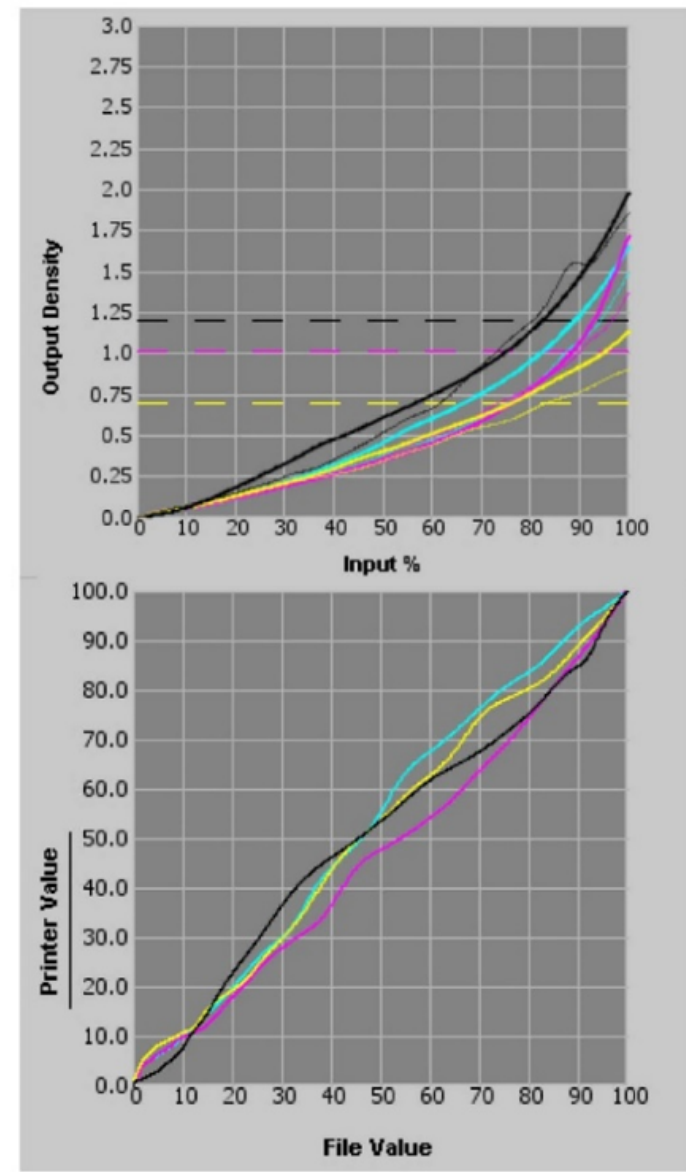

Measured Solid Ink Density (SID) of:

\begin{tabular}{|c|c|c|c|c|c|c|c|c|}
\hline \multicolumn{4}{|c|}{ AM Screening } & & \multicolumn{4}{|c|}{ FM Screening } \\
\hline $\begin{array}{l}C \\
1.45\end{array}$ & $\underset{1.36}{M}$ & $\begin{array}{l}Y \\
0.90\end{array}$ & $\begin{array}{l}K \\
1.73\end{array}$ & vs. & $\begin{array}{l}C \\
1.49\end{array}$ & $\begin{array}{l}M \\
1.37\end{array}$ & $\begin{array}{c}Y \\
0.90\end{array}$ & $\begin{array}{c}K \\
1.85\end{array}$ \\
\hline
\end{tabular}

» Figure 4: Calibration of AM vs. FM Screening

photometer with an $\mathrm{i} 1 \mathrm{iO}$ table and the data were run through this application. The printer profile was created and stored at the right location on the computer. The profile format version is 4.00 and it is considered as the Output Device Profile (ODP) of AM screening. This profile was used as a destination profile (DP) in the workflow. The source profile (SP) used in the experiment is a GRACoL2013 for characterized reference printing conditions-6 (CRPC-6). The same procedures as described above in this section were followed for creating the ODP for FM screening with an FM screened calibration curve and the $600 \mathrm{DPI}$ as the printer resolution. See Figure 6 for all output device profiles comparison (GRACoL2013, AM vs FM Screened) with profile volume (gamut volume) and $L^{*} a^{*} b^{*}$ values of each profile used. 


\section{Printing Samples for the Analysis}

Each COMA in the experiment (see Table 2) was considered as a group. A group involves a set of print parameters, such as: a digital halftone screening technique [amplitude modulate (AM) $\underline{\text { OR frequency modulate }}$ (FM)], the calibration curve (of AM OR FM screened), a color source profile [General Requirements for Applications in Commercial offset Lithography for characterized reference printing conditions-6 (GRACoL2013 for CRPC-6)], and a color destination profile of a digital press (AM OR FM screened). As listed below in the table 2 and as parameters illustrated in the figure 5, test target was printed for use in the experiment. A total of 100 sheets/samples were printed for each COMA by enabling the color management technique at the RIP. The digital press AM or FM calibration curve, AM or FM screening destination profile, and the source profiles all were applied during the printing (see Figure 5).

A total of 800 prints (copies) were printed, 100 for each COMA or COS with the same target image on a $100 \mathrm{LB}$ matte-coated paper $\left(K=8, N=800, n_{i}=80, n_{t}=640\right)$. For each group, 80 prints ( $n=80$ at $95 \%$ confidence level) were randomly pulled (or selected) for data collection/analysis. A total of 640 prints were analyzed $\left(n_{t}\right.$ $=640$ ). Colorimetric data for various color quantification for each group were generated from the printed colors. This study was focused on the measurement of color prints, printed with multiple COMA (See Table 2 \& Figures 3, 3A, \& 5) to identify the significant differences that exist among the multiple COMA. The type of paper used for the printing will have a significant impact on the print attributes, in turn they affect the print quality/visual appearance of colors (hue). Quality of printed halftone image is influenced by the print parameters and attributes (device profiles, screening techniques, resolution, and calibration data) which are part of the workflow. The print technology of interest for this study is dry-toner color electrophotography.

Upon printing the test images, multiple types of International Color Consortium (ICC) standard based color management applications (software) and instruments/ devices (Spectro-photometer \& Densitomter) were used for the data collection. The data were extracted by using an X-Rite Eye-One Spectrophotometer from the color printed samples for the statistical analysis to determine the significant differences that exist among the eight different COMA color attributes. Print/Color attributes (primary colors and gray hue) from each group were analyzed/compared with one another. Colorimetric data were collected from the ISO 12647 (2013) CMYK control strip. Statistical Package for Social Sciences (SPSS) was used to analyze the collected data to determine the colorimetric variation (COLVA) among the multiple

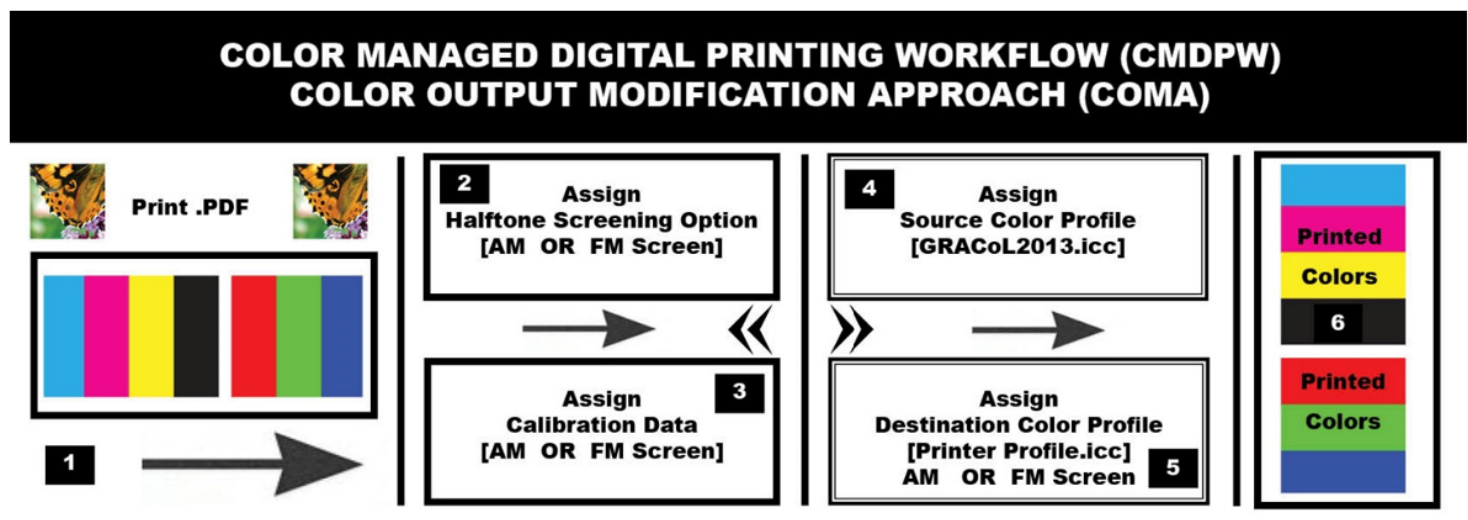

» Figure 5: Schematic Illustration of COMA

Table 2

Color Output (print) Modification Approaches (COMA) in a Color Managed Workflow (CMW)

\begin{tabular}{l|l|l|l|l}
\hline $\begin{array}{l}\text { COMA } \\
\text { Group }=\mathbf{K}\end{array}$ & $\begin{array}{l}\text { Type of } \\
\text { Halftone Screen }\end{array}$ & $\begin{array}{l}\text { Destination Profile } \\
\text { (of Screened) }\end{array}$ & $\begin{array}{l}\text { Calibration } \\
\text { (of Screened) }\end{array}$ & $\begin{array}{l}\text { Source } \\
\text { Profile }\end{array}$ \\
\hline 1 & AM & AM & AM & GRACoL2013.icc \\
\hline 2 & FM & FM & FM & GRACoL2013 \\
\hline 3 & AM & FM & FM & GRACoL2013 \\
\hline 4 & AM & FM & AM & GRACoL2013 \\
\hline 5 & FM & FM & AM & GRACoL2013 \\
\hline 7 & FM & AM & AM & GRACoL2013 \\
\hline 8 & FM & AM & FM & GRACoL2013 \\
\hline & AM & AM & FM & GRACoL2013 \\
\hline
\end{tabular}




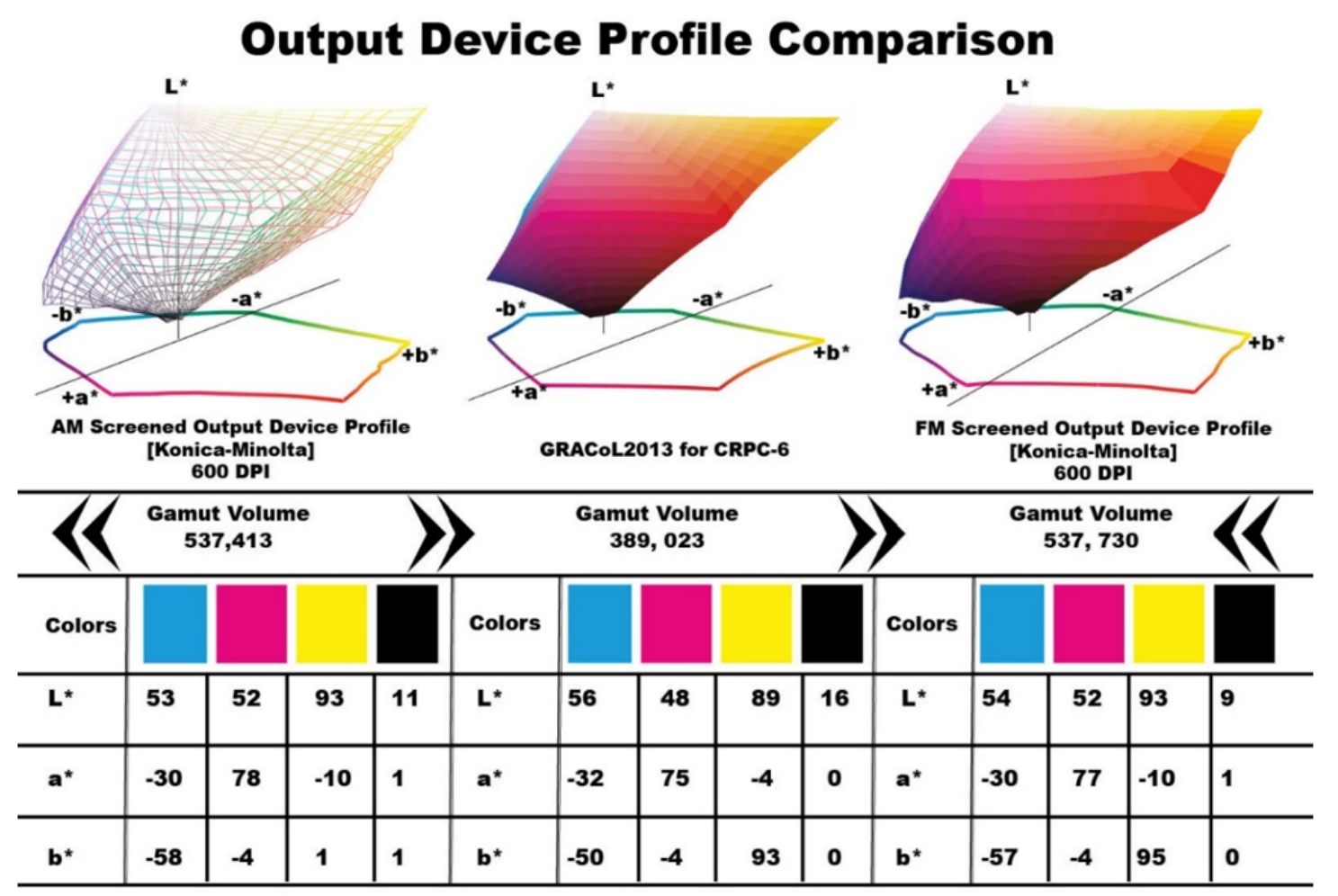

» Figure 6: Output Device Profiles Comparison

COMA groups. Since $K=8$, Analysis of Variance (ANOVA) with equal n's method (at acceptable margin of error $\alpha$ $=0.05)$ inferential statistics was used to determine the significant differences that exist among the $(K=8, N=$ $800, n_{i}=80, n_{t}=640$ ) groups mean color deviations of the various COMA groups (Glass, \& Hopkins, 1996).

\section{Data analysis \& research findings}

The descriptive and inferential statistical methods were used to analyze the data and presented in the following pages/tables. Subjective judgment on color difference/ deviation was not used in this study. The subjective judgment of color difference could differ from person to person. For example, people see colors in an image not by isolating one or two colors at a time (Goodhard \& Wilhelm, 2003), but by mentally processing contextual relationships between colors where the changes in lightness (value), hue, and chroma (saturation) contribute independently to the visual detection of spatial patterns in the image (Goodhard \& Wilhelm, 2003). Instruments, such as colorimeters and spectrophotometers, could eliminate the subjective errors of color evaluation perceived by human beings. In comparing the color differences between two colors, a higher $(\Delta \mathrm{E}$ or $\Delta \mathrm{H})$ is an indication that there is more color difference and a lesser $(\Delta \mathrm{E}$ or $\Delta \mathrm{H})$ is an indication of less color difference.

Statistical Package for Social Sciences (SPSS) was used to analyze the collected data to determine the colo- rimetric variation (COLVA) among the eight groups. Since the $K=8$, a one-way Analysis of Variance (ANO$V A$ ) with equal n's method (at $\alpha=0.05$ ) was used to determine the significant differences that exist among the $\left(K=8, n_{i}=80, n_{t}=640\right)$ group means (averages) color deviations of the various COMA (Glass \& Hopkins, 1996). The F-test can be calculated by using the following equation (Glass \& Hopkins, 1996).

$$
F=\frac{\sigma_{b}^{2}}{\sigma_{w}^{2}}=\frac{M S_{b}}{M S_{w}}=\frac{S S_{b} / V_{b}}{S S_{w} / V_{w}}=\frac{\sum n_{k}\left(\bar{X}_{k}-\bar{X}\right)^{2} / K-1}{\sum\left(X_{i k}-\bar{X}_{k}\right)^{2} / N-K}
$$

When statistically significant effects were detected among the eight groups, the Tukey method- Post hoc ANOVA analysis was used to determine which group (K) means were significantly different. The Tukey method is also known as the honest significant difference (HSD) test between two sample means (averages) which can be determined by using the following equation (Glass \& Hopkins, 1996). The F distribution and a probability value $p$, which is derived from the $F$, were used to determine if significant differences exist in the output color attributes of COMA. $F$ is a ratio of two independent estimates of the variance of the sample, namely between the groups and within the groups $\left(K=8, n_{i}=80, n_{t}=\right.$ 640). A low $p$ value (or higher $F$ value) is an indication that one should reject the stated null hypothesis $\left(\mathrm{H}_{0}\right)$ in favor of the stated alternative hypotheses $\left(\mathrm{H}_{\mathrm{a}}\right)$. This is interpreted as one of the COMA means (average) is significantly different. It suggests that there is a strong 
support that at least one pair of the COMA group means (averages) is not equal. The higher the $p$ value (or lower $F$ value) indicates that the means of various color attributes of the COMA are not statistically different.

$$
q_{1}=\frac{\bar{X}_{1}-\bar{X}_{K}}{S_{\bar{X}}}
$$

The value of $q$ is the difference between the larger and smaller means of the two samples. Differences among the means at $p \leq 0.05$ were considered to be statistically significant among all the eight COMA groups. The main effect of the print parameters on the digital color output in a CMDPW was determined by using the above stated methods ( $F$ and $q)$. The HSD multiple comparison test (with $\alpha=0.05$ ) in the experiment that enabled the researcher to identify the significant difference between one COMA group to others. In other words, which COMA differs significantly from another?

Colorimetric, densitometric, and spectrophotometric computations were used to determine the gray color (overlap of $\mathrm{C}=50 \%, \mathrm{M}=40 \%$, and $\mathrm{Y}=40 \%$ ) hue variation $(\Delta \mathrm{H})$ among the eight COMA or COS. The data for each group were generated from the printed colors of the target (ISO 12647-7, 2013 Control Wedge/Strip) image (see Figure 7) by using an Eye-One-Pro spectrophotometer with interface application, such as the CGS-ORIS CertifiedWEB against the GRACoL2013_CRPC6 reference data. Measured colorimetric data (CIE L* $a^{*} b^{*}$ ) from ISO 12647-7 control strip were used to determine the means (averages) of: Gray $\Delta \mathrm{H}$ (CMY overlap) between the printed colors and its reference data (IT8.7/4). Data derived from ISO 12647-7 control strip (sample) is the difference between the characterization data set (full IT8.7/4 target) and the sample.

The control strip (wedge) image is intended primarily as a control device for pre-press proofs but may also be used to control production printers or presses. The wedge has 3 rows and 84 patches, and it contains only a small sub-sample of the total printable color gamut. The wedge contains too few patches to prove an accurate match to a specification like GRACoL or SWOP (Specifications for Web Offset Publications). It does contain enough patches to monitor the stability of a system that has previously been tested with a target such as the IT8.7/4 (CMYK target image). The reference file content for this image (IT8.7/4) are the CMYK dot percentage values and nominal $\mathrm{CIE} L^{*} a^{*} b^{*}$ characterization data values for the GRACoL2013-CRPC6 reference.

\section{Delta $E$ and Delta Hue ( $\Delta \mathrm{E}^{*}$ and $\left.\Delta \mathrm{H}^{*}\right)$}

The following equations are used by a spectrophotometer and the interface software to calculate the color variation between the printed samples and reference (GRACoL2013-CRPC6). Assessment of color is more than a numeric expression. It is an assessment of the difference in the color sensation (delta) from a known standard. In the CIELAB color model, two colors can be compared and differentiated. The expression for these color differences is expressed as $\Delta \mathrm{E}$ (Delta $\mathrm{E}$ or Difference in Color Sensation). The following equation is used to calculate the $\Delta E$ (Committee for Graphic Arts Technologies Standards, 2003).

$$
\Delta E^{*}=\sqrt{\left(L_{1}-L_{2}\right)^{2}+\left(a_{1}-a_{2}\right)^{2}+\left(b_{1}-b_{2}\right)^{2}}
$$

Where: $1=$ Reference Color and $2=$ Printed Color
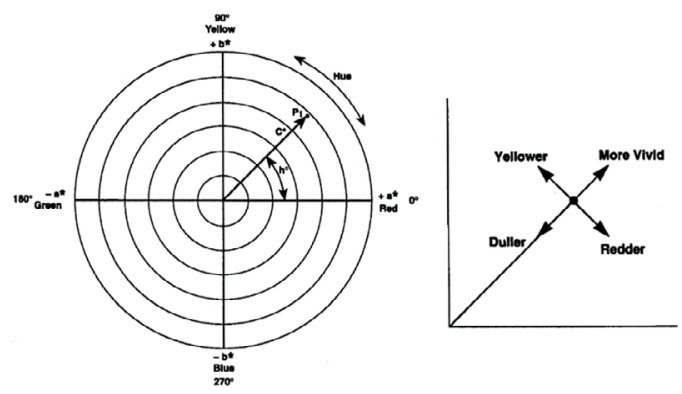

» Figure 8: Schematic of $L^{*} a^{*} b^{*} \& c^{*}, h^{*}$ Coordinates

Calculation of $\Delta H^{*}$ and $\Delta E^{*}$ requires colorimetric data from the $L^{*} a^{*} b^{*}$ model. Metric hue angle $h^{*}$ is defined by the following formulas (Morovic, 2002):
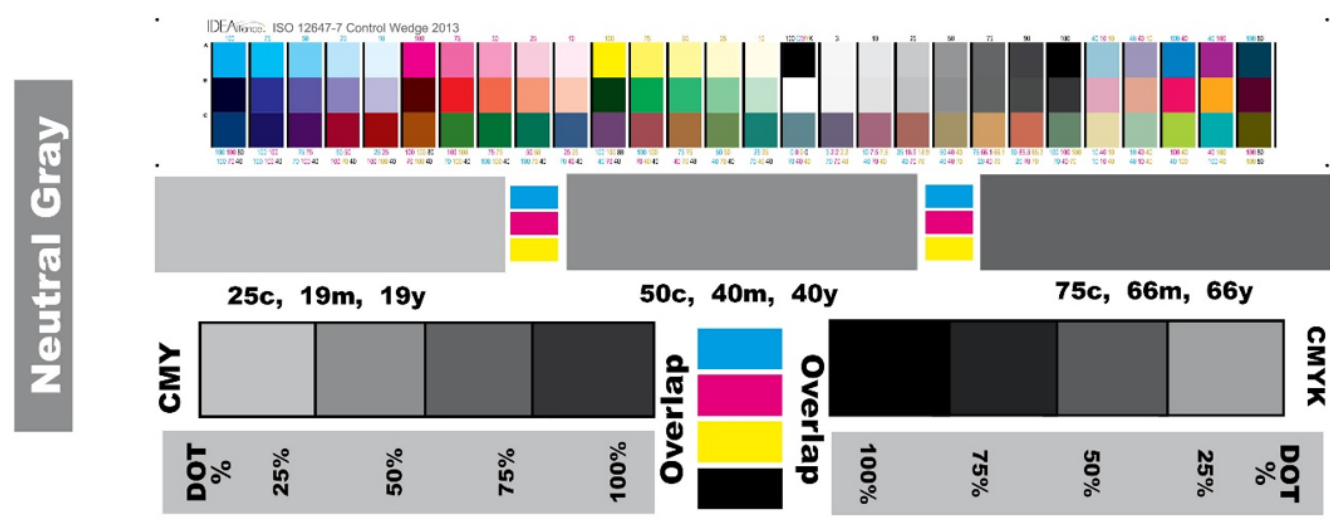

» Figure 7: ISO 12647-7, 2013 Control Wedge/Strip with additional Color Patches 
Metric hue angle: $h *_{a b}=\tan ^{-1}\left(\frac{b *}{a *}\right)$

Where: $a^{*}, b^{*}$ are chromaticity coordinates in $L^{*} a^{*} b^{*}$ color space

Hue difference $\left(\Delta H^{*}\right)$ is calculated by the following formula (Morovic, 2002):

$$
\begin{aligned}
\Delta H^{*} & =\sqrt{\left(\Delta E^{*} a b\right)^{2}-\left(\Delta L^{*}\right)^{2}-\left(\Delta C^{*}\right)^{2}} \\
& =\sqrt{\left(\Delta a^{*}\right)^{2}+\left(\Delta b^{*}\right)^{2}-\left(\Delta C^{*}\right)^{2}}
\end{aligned}
$$

(5) Colorimetric Variation (COLVA) of the Gray Balance (Gray Hue), Hypothesis: Gray Color (Overlap of CMY) Hue Deviation ( $\Delta \mathrm{H})$ : Reference vs. Printed Colorimetry

The ANOVA test revealed that there was a significant difference among the gray $\Delta \mathrm{H}$ produced by

(6) each (or multiple) COMA groups, $F(7,632)=722.116$, $p=0.000$. Therefore, established null hypothesis was rejected and alternative hypothesis was retained. Data indicated that each of the COMA group altered the printed gray colors differently.

Colorimetric Variation (COLVA) of Multiple COMA Groups Gray (CMY Overlap) Hue Deviation

\section{Color Deviation $\Delta \mathrm{E} / \Delta \mathrm{H}$}

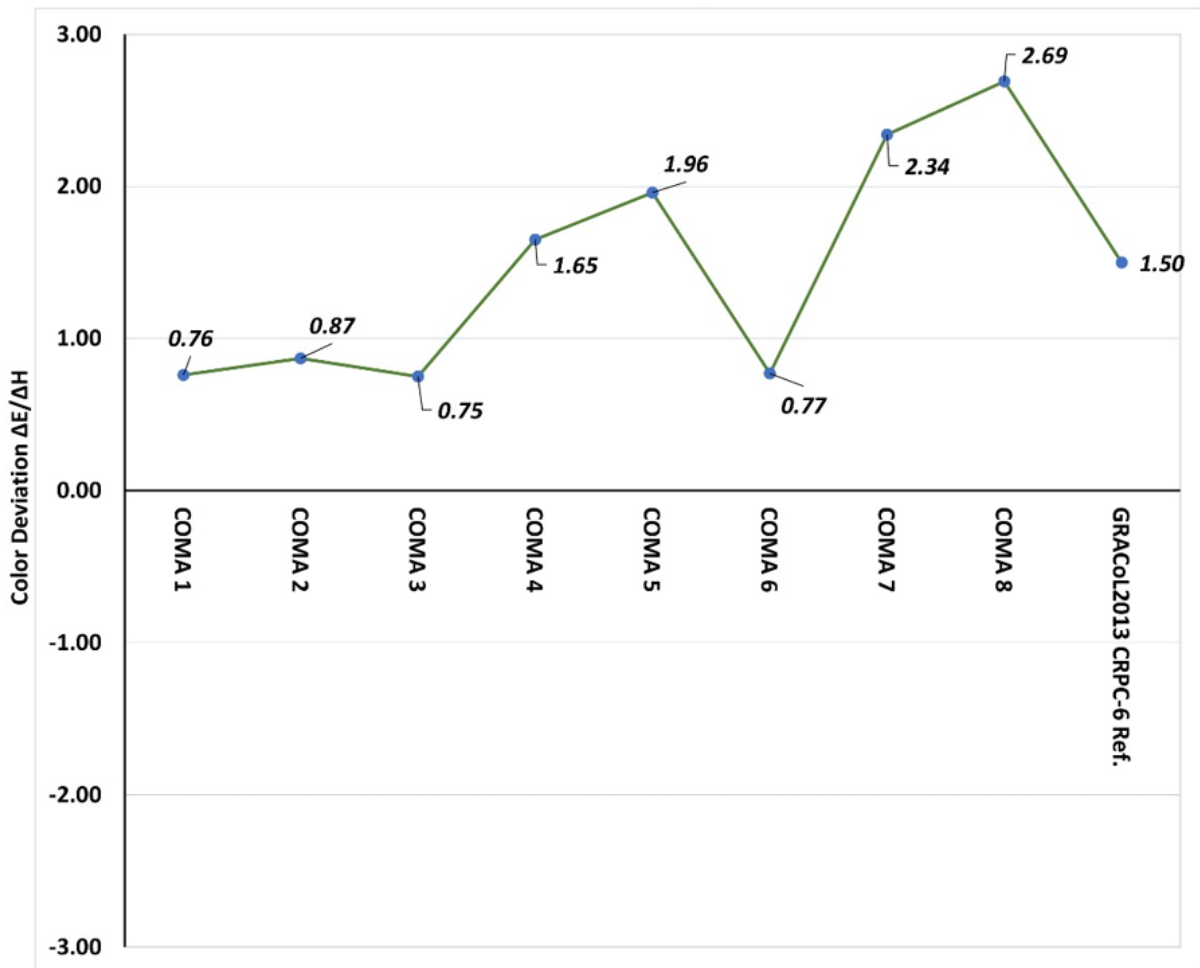

» Figure 9: Gray (Overlap of $C M Y$ ) Hue Deviation $(\Delta H)$ vs. GRACoL2013-CRPC 6 Gray $\triangle H$

Table 3

Summary of ANOVA for Multiple COMA Influence on the Gray $\Delta H$

\begin{tabular}{l|l|l|l|l|l}
\hline Source of Variation & Sum of Square & df & Mean Square & F & Sig. \\
\hline Between Groups & 140.439 & 7 & 20.063 & 722.116 & $0.000^{* *}$ \\
\hline Within Groups & 6.890 & 632 & 0.028 & & \\
\hline Total & 147.329 & 639 & & & \\
\hline
\end{tabular}

*Significant Difference $[(\alpha=0.05>0.001)(F=722.116<2.024)]$ 
This is due to applied print parameters (see Figure 9). As such, the effect was significant at the $p<0.05$ for all eight COMA (see Table 3). Post hoc analysis using the Tukey HSD criterion for significance among the multiple COMA groups $(\mathrm{K}=8)$ averages indicated that when comparing COMA 1, 2, 3, and 6 with other $\operatorname{COMA}(4,5,7$, and 8) groups, there was a significant statistical difference in the gray $\Delta \mathrm{H}$ produced by these COMA (see Table 4) at the $p \leq 0.05$. The Tukey HSD test also indicated that the mean scores of gray $\triangle \mathrm{H}$ of multiple COMA $(1,2,3$, and 6 vs. 4, 5, 7, and 8) are significantly different from each other. The COMA 8 resulted in producing the highest gray $\Delta \mathrm{H}$, while COMA 1 produced the lowest. No significant differences were found among gray $\Delta \mathrm{H}$ mean scores of COMA 1, 2, 3, and 6 (See Table 4). Significant differences were found among COMA 4, 5, 7, and 8 when comparing with COMA 1, 2, 3, and 6 (see Figure 10).

Table 4

Tukey HSD Multiple Comparison of COMA Influence on the Gray $\Delta \mathrm{H}$

\begin{tabular}{l|l|l|l}
\hline COMA & $\begin{array}{l}\text { Mean } \\
\text { Difference }\end{array}$ & $\begin{array}{l}\text { Standard } \\
\text { Deviation (SD) } \\
\text { Difference } \\
\mathbf{n}=\mathbf{8 0}\end{array}$ & Sig. \\
\hline 1 vs.2 & -0.109 & -0.09 & 0.155 \\
\hline 3 & 0.017 & 0.016 & 1.000 \\
\hline 4 & -0.996 & 0.045 & $0.000^{* *}$ \\
\hline 5 & -1.195 & 0.053 & $0.000^{* *}$ \\
\hline 6 & -0.005 & 0.0071 & 1.000 \\
\hline 7 & -1.580 & -0.001 & $0.000^{* *}$ \\
\hline 8 & -1.924 & -0.002 & $0.000^{* *}$ \\
\hline$* p \leq 0.05$ and $* * p \leq 0.001$ & {$\left[\mathbf{K}=\mathbf{8}, \mathbf{N}=\mathbf{8 0 0}, \mathbf{n}_{\mathbf{i}}=\mathbf{8 0}, \mathbf{n}_{\mathbf{t}}=\mathbf{6 4 0}\right]$}
\end{tabular}

\section{Visual Comparison of Average Colorimetric Variation (COLVA) of Gray Hue among the Multiple COMA Groups, $\mathrm{K}=8$}

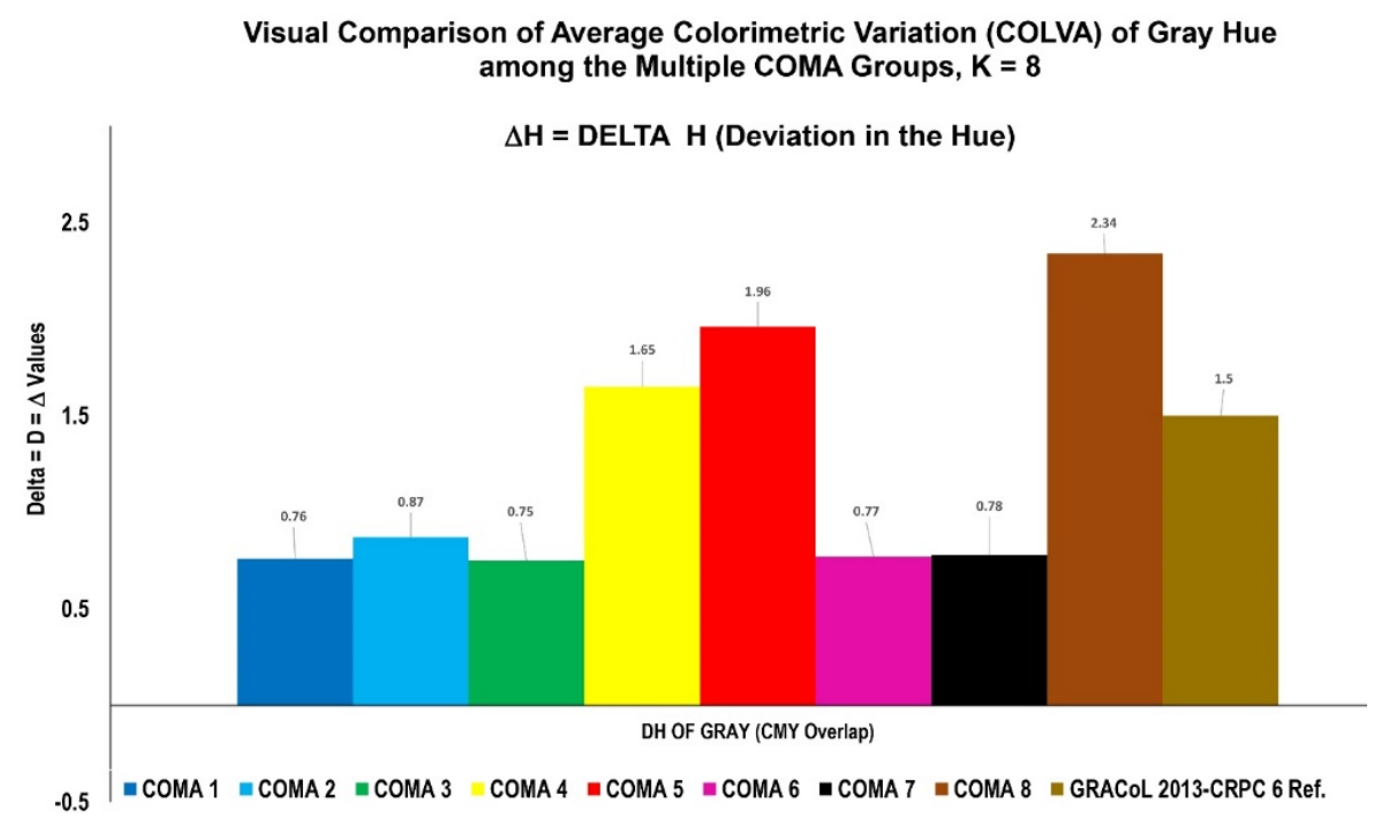

» Figure 10: Average Gray Hue Deviation among the Multiple COMA vs. GRACoL2O13 Ref.

[Colors presented in this chart do not represent actual COMA group produced colors]
This research demonstrated the use of Analysis of Variance (ANOVA) to determine the effect of Color Output Modification Approach (COMA) or Color Output Sequences (COS) in a Color Management Digital Printing Workflow (CMDPW). Correct COMA approach would be assigning the correct print parameters during the printing. Print parameters play an important role in a CMDPW. This is as per the color science of a Color Managed Workflow (CMW), which in turn relies on the 4-C's (Calibration, Characterization, Conversion, and the Control). The findings of this study represent specific printing or testing conditions. The images, printer, instrument, software, and paper that were utilized are important factors to consider when evaluating the results. The findings of the study cannot be generalized to other CMDPW. However, other graphic arts educators, industry professionals, and researchers may find this study meaningful and useful. For example, educators can implement similar or the presented model (or method) to teach color management concepts.

For each COMA, colorimetric variation (COLVA) in the gray balance was determined on the basis of GRACoL2013-CRPC 6 reference. The data from the ANO$V A$ test revealed that there were significant differences in the color reproduction among multiple COMA. The printed gray average color (overlap of $\mathrm{CMY}$ ) $\triangle \mathrm{H}$ of the multiple COMA groups ( $N=8$, Average $\Delta H=1.47$ ) was found to be within the GRACoL2013-CRPC reference $(\Delta H=1.50)$. It suggests that a mismatch of print parameters within the COMA groups did not affect the gray hue deviation. However, the ANOVA test revealed that significant differences exists in the gray hue among the multiple COMA or COS. 
As a result of this experiment, it is evident that color management works and it offers more flexibility and control over color reproduction images. The findings determined that only the optical aspects of color are quantitatively analyzable and measurable because humans perceive color subjectively. It will be hard to document and measure the color values we see or detect. Additionally, the implementation of CMDPW is costly, time consuming and a tedious process. It does, however, benefit those who implement this workflow to get consistent color from device to device. Future study is needed to determine the gray hue of color management workflow vs. non-color management workflow. This study was limited to the electrophotographic printing process (color laser) only. Future studies can be conducted by using other printing processes. The colorimetric data of this experiment led to the conclusion that the application of a correct print parameters set-up is an important step in a CMDPW in order to output accurate colors or gray balance of choice for a desired use/purpose. Mismatch of print parameters could result in a color management discrepancy.

\section{References}

Chung, R. \& Ma, L. (1995) Press Performance Comparison between AM and FM Screening. TAGA Proceedings 1995. New York, TAGA.

Committee for Graphic Arts Technologies Standards. (2003) ANSI/CGATS.5-2003. Graphic technologyspectral measurement and colorimetric computation for graphic arts image. Reston, The Association for Suppliers of Printing and Publishing Technologies.
Fleming, P., Dollak, J. \& Fryzlewicz, S. (2008) Stochastic Screening: What to do When Your Rip Doesn't Support It and Comparison with Conventional Screening on an Offset Press. Journal of Graphic Technology. 1 (3), 15-23

Glass, G. V., \& Hopkins, K. D. (1996) Statistical Methods in Education \& Psychology. Boston, MA: Allyn \& Bacon.

Goodhard, M. M. \& Wilhelm, H. (2003). A new test method based on CIELAB colorimetry for evaluating the permanence of pictorial images. Available from: http://www.wilhelm-research.com/pdf/WIR_CIELAB_ TEST_2003_07_25.pdf [Accessed 20th August 2019]

Ma, L.L. (2003) Understanding Digital Halftones. Journal of Visual Communications. 130-136.

Morovic, J. (2002) Colour gamut mapping. In: Green, P. \& MacDonald, L. (eds.) Colour Engineering Achieving Device Independent Colour. New York, John Wiley \& Sons, pp. 297-314.

Novaković, D. \& Avramović, D. (2012) Influence of Printing Surface Attributes on Print Quality in Electrophotography. Technical Gazette. 19 (2), 295-301.

Pnueli, Y. \& Bruckstein, A. (1996) Gridless Halftoning: A Reincarnation of the Old Method. Graphical Models and Image Processing. 58 (1), 38-64. Available from: doi: 10.1006/gmip.1996.0003

Wales, T (2009) Paper: The Fifth Color. In: IPA Bulletin. Alexandria, IDEAlliance.

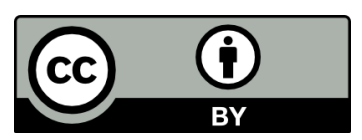

(C) 2020 Authors. Published by the University of Novi Sad, Faculty of Technical Sciences, Department of Graphic Engineering and Design. This article is an open access article distributed under the terms and conditions of the Creative Commons Attribution license 3.0 Serbia (http://creativecommons.org/licenses/by/3.0/rs/). 